\title{
BMJ Open SUGAR-DIP trial: oral medication strategy versus insulin for diabetes in pregnancy, study protocol for a multicentre, open-label, non-inferiority, randomised controlled trial
}

Leon de Wit, ${ }^{1}{ }^{1}$ Doortje Rademaker, ${ }^{2}$ Daphne N Voormolen, ${ }^{1}$ Bettina M C Akerboom, ${ }^{3}$ Rosalie M Kiewiet-Kemper, ${ }^{4}$ Maarten R Soeters, ${ }^{5}$ Marion A L Verwij-Didden, ${ }^{6}$ Fahima Assouiki, ${ }^{7}$ Daniela H Schippers, ${ }^{8}$ Mechteld A R Vermeulen, ${ }^{9}$ Simone M I Kuppens, ${ }^{10}$ Mirjam M Oosterwerff, ${ }^{11}$ Joost J Zwart, ${ }^{12}$ Mattheus J M Diekman, ${ }^{13}$ Tatjana E Vogelvang,,${ }^{14} \mathrm{P}$ Rob J Gallas, ${ }^{15}$ Sander Galjaard, ${ }^{16}$ Willy Visser, ${ }^{16}$ Nicole Horree, ${ }^{17}$ Tamira K Klooker, ${ }^{18}$ Rosemarie Laan, ${ }^{19}$ Rik Heijligenberg, ${ }^{20}$ Anjoke J M Huisjes, ${ }^{21}$ Thomas van Bemmel, ${ }^{22}$ Claudia A van Meir, ${ }^{23}$ Annewieke W van den Beld, ${ }^{24}$ Wietske Hermes ${ }^{25}$ Solrun Vidarsdottir, ${ }^{26}$ Anneke G Veldhuis-Vlug, ${ }^{27}$ Remke C Dullemond, ${ }^{28}$ Henrique J Jansen, ${ }^{29}$ Marieke Sueters, ${ }^{30}$ Eelco J P de Koning, ${ }^{31}$ Judith O E H van Laar, ${ }^{32}$ Pleun Wouters-van Poppel, ${ }^{33}$ Marina E Sanson-van Praag, ${ }^{34}$ Eline S van den Akker, ${ }^{35}$ Catherine B Brouwer, ${ }^{36}$ Brenda B Hermsen, ${ }^{35}$ Bert Jan Potter van Loon, ${ }^{36}$ Olivier W H van der Heijden, ${ }^{37}$ Bastiaan E de Galan, ${ }^{38}$ Marsha van Leeuwen, ${ }^{39}$ Johanna A M Wijbenga, ${ }^{40}$

To cite: de Wit L, Rademaker D, Voormolen DN, et al. SUGAR-DIP trial: oral medication strategy versus insulin for diabetes in pregnancy, study protocol for a multicentre, open-label, non-inferiority, randomised controlled trial. BMJ Open 2019;9:e029808. doi:10.1136/ bmjopen-2019-029808

- Prepublication history and additional material for this paper are available online. To view these files, please visit the journal online (http://dx.doi. org/10.1136/bmjopen-2019029808).

Received 12 February 2019 Revised 11 April 2019 Accepted 22 May 2019

Check for updates

(C) Author(s) (or their employer(s)) 2019. Re-use permitted under CC BY-NC. No commercial re-use. See rights and permissions. Published by BMJ.

For numbered affiliations see end of article.

Correspondence to

Dr. Bas B van Rijn;

b.vanrijn@erasmusmc.nl

Karin de Boer, ${ }^{41}$ Arianne $\mathrm{C}$ van Bon ${ }^{42}$ Flip W van der Made, ${ }^{43}$ Silvia A Eskes, ${ }^{44}$ Mirjam Zandstra, ${ }^{45}$ William $\mathrm{H}$ van Houtum, ${ }^{46}$ Babette A M Braams-Lisman, ${ }^{47}$ Catharina R G M Daemen-Gubbels, ${ }^{48}$ Maurice G A J Wouters, ${ }^{49}$ Richard G IJzerman, ${ }^{50}$ Nico A Mensing van Charante, ${ }^{51}$ Rolf Zwertbroek, ${ }^{52}$ Judith E Bosmans, ${ }^{53}$ Inge M Evers, ${ }^{54}$ Ben Willem Mol, ${ }^{55}$ Harold W de Valk, ${ }^{56}$ Floris Groenendaal, ${ }^{57}$ Christiana A Naaktgeboren, ${ }^{58}$ Rebecca C Painter, ${ }^{2}$ $\mathrm{J}$ Hans deVries, ${ }^{59}$ Arie Franx, ${ }^{1}$ Bas B van Rijn ${ }^{1,60}$

\section{ABSTRACT}

Introduction In women with gestational diabetes mellitus (GDM) requiring pharmacotherapy, insulin was the established first-line treatment. More recently, oral glucose lowering drugs (OGLDs) have gained popularity as a patient-friendly, less expensive and safe alternative. Monotherapy with metformin or glibenclamide (glyburide) is incorporated in several international guidelines. In women who do not reach sufficient glucose control with OGLD monotherapy, usually insulin is added, either with or without continuation of OGLDs. No reliable data from clinical trials, however, are available on the effectiveness of a treatment strategy using all three agents, metformin, glibenclamide and insulin, in a stepwise approach, compared with insulin-only therapy for improving pregnancy outcomes. In this trial, we aim to assess the clinical effectiveness, cost-effectiveness and patient experience of a stepwise combined OGLD treatment protocol, compared with conventional insulin-based therapy for GDM.

Methods The SUGAR-DIP trial is an open-label, multicentre randomised controlled non-inferiority trial. Participants are women with GDM who do not reach target glycaemic control with modification of diet, between 16 and 34 weeks of gestation. Participants will be randomised to either treatment with OGLDs, starting with metformin and supplemented as needed with glibenclamide, or randomised to treatment with insulin. In women who do not reach target glycaemic control with combined metformin and glibenclamide, glibenclamide will be substituted with insulin, while continuing metformin. The primary outcome will be the incidence of large-forgestational-age infants (birth weight $>90$ th percentile). Secondary outcome measures are maternal diabetesrelated endpoints, obstetric complications, neonatal complications and cost-effectiveness analysis. Outcomes will be analysed according to the intention-to-treat principle.

Ethics and dissemination The study protocol was approved by the Ethics Committee of the Utrecht University Medical Centre. Approval by the boards of management for all participating hospitals will be obtained. Trial results will be submitted for publication in peer-reviewed journals. Trial registration number NTR6134; Pre-results. 


\section{INTRODUCTION}

The prevalence of gestational diabetes mellitus (GDM) is rising and currently affects approximately 1\%-28\% of all pregnancies, varying by region and diagnostic criteria used. ${ }^{1-4}$ GDM carries significant perinatal risks for pregnancy and childbirth, such as polyhydramnios, small-for-gestational-age and large-for-gestational-age infants, macrosomia, stillbirth, shoulder dystocia, obstructed labour, pre-eclampsia (PE) and neonatal hypoglycaemia. ${ }^{5-9}$ In addition, increasing concern exists about the impact of GDM on offspring development and associated long-term risks for glucose and insulin resistance, obesity and chronic disease in children born to mothers with GDM. ${ }^{10-12}$

The rising number of women diagnosed with GDM is increasingly putting pressure on healthcare resources. Effective treatment for GDM treatment requires a multidisciplinary approach by midwives, obstetricians, dieticians, endocrinologists and diabetes nurse specialists. Current treatment of GDM focuses on achieving optimal glycaemic control. When blood glucose levels, usually based on self-monitoring, fall outside the target range despite lifestyle and dietary advice, treatment with antihyperglycaemic medication is indicated. ${ }^{13} 14$ As pharmacological treatment, subcutaneous insulin injections have traditionally been used as first-choice treatment for GDM and is still advocated in many, ${ }^{15-18}$ but not all guidelines. ${ }^{19-21}$ In recent years, clinical research and experience with oral glucose lowering drugs (OGLDs) has shown promising results as a treatment alternative that may substitute insulin in many women. ${ }^{22} 23$

Metformin and glibenclamide (glyburide) are the OGLDs most studied for diabetes in pregnancy. Both are already widely used in the treatment of GDM, considered to be safe and have been incorporated in several guidelines as treatment options alongside insulin. ${ }^{19-21} 2425 \mathrm{~A}$ 2014 retrospective cohort study from the USA showed that the use of glibenclamide had increased from $7.4 \%$ in 2000 to $64.5 \%$ in 2011 , becoming the most common treatment for GDM requiring pharmacotherapy in $2007 .{ }^{26} \mathrm{In}$ the UK, incorporated in NICE guidelines (National Institute for Health and Care Excellence, UK), metformin is the first-choice treatment, supplemented with insulin if needed. ${ }^{19}$ Insulin is offered to women if metformin is contraindicated or unacceptable to the patient, or target glucose values are not met with metformin only. NICE guidelines state that glibenclamide could be considered an option for women in whom blood glucose targets are not achieved with metformin, but decline insulin therapy, or for those who cannot tolerate metformin. The International Federation of Gynaecology and Obstetrics and more recently the Society of Maternal-Fetal Medicine Committee further endorsed OGLDs as a reasonable and safe first-line pharmacological treatment option in GDM, with metformin being preferred over glibenclamide. ${ }^{21} 25$ In contrast, in the Netherlands, insulin has remained the drug of choice in the majority of hospitals.
Strengths and limitations of this study

- This is the first open-label randomised controlled trial that directly compares a stepwise treatment protocol using a combination of oral glucose lowering drugs (OGLDs) with insulin as a first-line treatment for gestational diabetes mellitus (GDM) not responding to diet.

- The randomised multicentre design minimises the risk of bias and increases generalisability of the results.

- Variation in diagnostic thresholds and treatment targets for GDM may need to be addressed to assess the value of this strategy across different populations.

Two 2017 Cochrane Reviews on 11 and 53 studies (1487 and 7381 women) concluded that due to insufficient high-quality evidence, no single agent is superior in the treatment of GDM. ${ }^{27}{ }^{28}$ And although the use of OGLDs is widespread, there is an ongoing discussion on which drug should be first-line treatment after lifestyle and dietary interventions. ${ }^{24}$ Both insulin and oral agents have advantages and disadvantages. Insulin is safe and effective; however, it is considered burdensome by pregnant women, requires intensive glucose monitoring and is associated with episodes of maternal hypoglycaemia. ${ }^{29}$ OGLDs are less costly, less burdensome and associated with higher patient satisfaction. ${ }^{23}$ 30-33 Metformin has the advantage over insulin that hypoglycaemic events do not occur, but it is less potent when compared with glibenclamide, can cause gastrointestinal side effects and is possibly associated with more spontaneous preterm deliveries. ${ }^{34}$ Glibenclamide, similar to insulin, is more potent in its glucose-lowering effect and may cause hypoglycaemia in the mother and newborn. ${ }^{22}{ }^{35}$ Other undesirable effects include gastrointestinal reactions, allergic skin reactions, altered liver enzyme values, visual disturbances and weight gain. And although intrauterine exposure to metformin or glibenclamide is not associated with congenital anomalies, much less is known about direct fetal metabolic effects and long-term effects on mothers and offspring. ${ }^{36}$

With current OGLD monotherapy, consisting of either metformin or glibenclamide, in women who do not reach glycaemic control, prompting the need for additional measures, in general OGLDs are replaced by or supplemented with insulin. A combination of oral agents may be an interesting strategy for GDM treatment; however, current evidence is insufficient to determine the optimal use of OGLDs. In a recent randomised controlled trial (RCT) by Nachum et al in 104 women with GDM, powered for glycaemic control, combination therapy of metformin and glibenclamide decreased the need for additional insulin from $32 \%$ to $11 \%$ ( $\mathrm{p}=0.0002$ ) compared with monotherapy. ${ }^{37}$ Metformin as the firstline therapy combined with glibenclamide if needed was associated with the highest treatment success. These data support the need for a well-powered large-scale RCT to compare a stepwise approach combining metformin and 
glibenclamide to conventional insulin therapy to study effects on pregnancy outcomes.

In the SUGAR-DIP trial, a multicentre RCT, we aim to assess non-inferiority of treatment with metformin, and in case of insufficient glycaemic control the addition of glibenclamide, compared with immediate insulin in the treatment of GDM. We expect that a proportion of patients will achieve glycaemic control with metformin only. By adding glibenclamide in combined treatment with metformin, we expect to achieve glycaemic control as good as by insulin, while maintaining the benefits and ease of a less burdensome treatment with oral medication. We will assess the clinical effectiveness, cost-effectiveness and patient experience of stepwise oral antihyperglycaemic medication to treat GDM compared with conventional insulin-based treatment strategy.

\section{METHODS}

\section{Design and setting}

The SUGAR-DIP trial is a multicentre non-inferiority RCT. The study will be open label as oral drugs and insulin cannot be administered individually in a blinded way. The study will be conducted within the setting of the Dutch Consortium for Healthcare Evaluation and Research in Obstetrics and Gynaecology-NVOG Consortium 2.0, ${ }^{38} \mathrm{a}$ collaborative network of all major hospitals in the Netherlands and the Dutch Society of Obstetrics and Gynaecology (NVOG) and performed by treatment teams generally consisting of an internal medicine specialist, a gynaecologist and diabetes nurses. The trial was approved by the Medical Research Ethics Committee (MREC) of the UMC Utrecht. Trial reference number: 16-523/M. The trial is registered in the Netherlands Trial Registry on 29 November 2016 under the number NTR6134. ${ }^{39}$

\section{Patient and public involvement}

In the preparation of the trial, the patient organisation Dutch Diabetes Association (Diabetes Vereniging Nederland) was involved. A questionnaire on patient perspectives of women who have (had) GDM was issued by the organisation prior to the development of the study protocol. The organisation was furthermore involved in reviewing the study protocol and provided valuable input in the development of the information material used in the study. On completion of the trial, the patient organisation will be involved in dissemination of the study results.

\section{Participants and eligibility criteria}

Women diagnosed with GDM who have not reached target glycaemic control with dietary and lifestyle adaptations and thus meet the criteria for additional treatment with antihyperglycaemic medication between 16 and 34 weeks of gestation will be eligible for inclusion. Target glycaemic control is defined by the NVOG (Dutch Society $\mathrm{O} \& \mathrm{G})$ diabetes in pregnancy guideline as a fasting glucose concentration $\leq 5.3 \mathrm{mmol} / \mathrm{L}$, 1 -hour postprandial $\leq 7.8 \mathrm{mmol} / \mathrm{L}$ or 2 -hour postprandial $\leq 6.7 \mathrm{mmol} / \mathrm{L}^{18}$
The diagnosis of GDM is made according to Dutch national guidelines, using a 75-gram oral glucose tolerance test (OGTT). ${ }^{18}$ Due to a transition in diagnostic thresholds, both the WHO 1999 (fasting $\geq 7.0 \mathrm{mmol} / \mathrm{L}$ or 2-hour postload $\geq 7.8 \mathrm{mmol} / \mathrm{L}$ ) and the WHO 2013 criteria (fasting $\geq 5.1 \mathrm{mmol} / \mathrm{L}$, 1-hour postload $\geq 10.0$ or 2-hour postload $\geq 8.5 \mathrm{mmol} / \mathrm{L}$ ) for venous plasma glucose values were used to diagnose GDM. The 100-gram OGTT is incorporated in the study protocol, as it is part of the Dutch national guideline; however, this test is not commonly used in the Netherlands. Although thresholds for the diagnosis of GDM in the Netherlands and therefore in the trial are divergent to some extent, the target glucose values to define insufficient glycaemic control (while on diet) as the additional inclusion criterion for enrolment in the trial apply to all centres. It is thus expected that patients eligible for enrolment form a homogeneous group despite differences in screening tools.

Screening in the Netherlands is conducted according to a high risk strategy and takes place in the second trimester (24-28 weeks) among pregnant women with one or more of the following risk factors: a history of GDM, body mass index $(\mathrm{BMI})>30\left(\mathrm{~kg} / \mathrm{m}^{2}\right)$, a history of a neonate with a birth weight $>95$ th percentile or $>4500$ grams, a first degree family member with diabetes, polycystic ovary syndrome, a history of an unexplained intrauterine death or an ethnicity with higher diabetes risk (eg, women from South Asia, Indian descent/Surinamese, Afro-Caribbean, Middle-Eastern, Moroccan or Egyptian ethnicity). In case of a history of GDM in a previous pregnancy, an OGTT as early as 16 weeks of gestation is recommended, to be repeated at 24-28 weeks if normal. An OGTT may furthermore be performed in case of suspected fetal macrosomia, polyhydramnios or symptoms of polydipsia or polyuria, also in women without risk factors.

For the SUGAR-DIP trial, we have set the upper limit for inclusion to 34 weeks, in line with previous trials, ${ }^{22} 2340$ allowing at least 4 weeks of exposure to pharmacological treatment. With the timing of the OGTT in current guidelines, it is expected that the majority of women will be treated for over 8 weeks. Although in women diagnosed later in pregnancy exposure to treatment may have less of an effect on the primary outcome, treatment may still influence several important secondary outcomes, such as neonatal hypoglycaemia.

Additional inclusion criteria for the SUGAR-DIP trial are (1) maternal age $\geq 18$ years, (2) singleton pregnancy, (3) ability to understand the Dutch or English language and (4) ability to provide written informed consent. Patients who meet any of the following criteria are excluded from the study: (1) known pre-existing type 1 or type 2 diabetes mellitus, (2) severe medical or psychiatric comorbidities, (3) significant liver disease or renal insufficiency, or any other known condition with contraindications for the use of either metformin or glibenclamide and (4) pregnancy with a fetus affected by major congenital birth defects and/or chromosomal abnormality. 


\section{Recruitment and randomisation}

Eligible women will be informed and invited to participate by either their diabetes care or obstetric care provider, that is, physician, obstetrician, midwife or diabetes nurse. Following counselling, written informed consent is obtained and participants are individually randomised to either stepwise OGLDs or insulin. Randomisation is performed through a central web-based tool (Castor
EDC, Ciwit B.V., Amsterdam, the Netherlands) using a 1:1 ratio and block randomisation with a variable block size of 4 and 6 .

\section{Intervention and control}

The stepwise treatment strategy for the intervention (OLGD) and control (insulin) group is displayed in figure 1 .

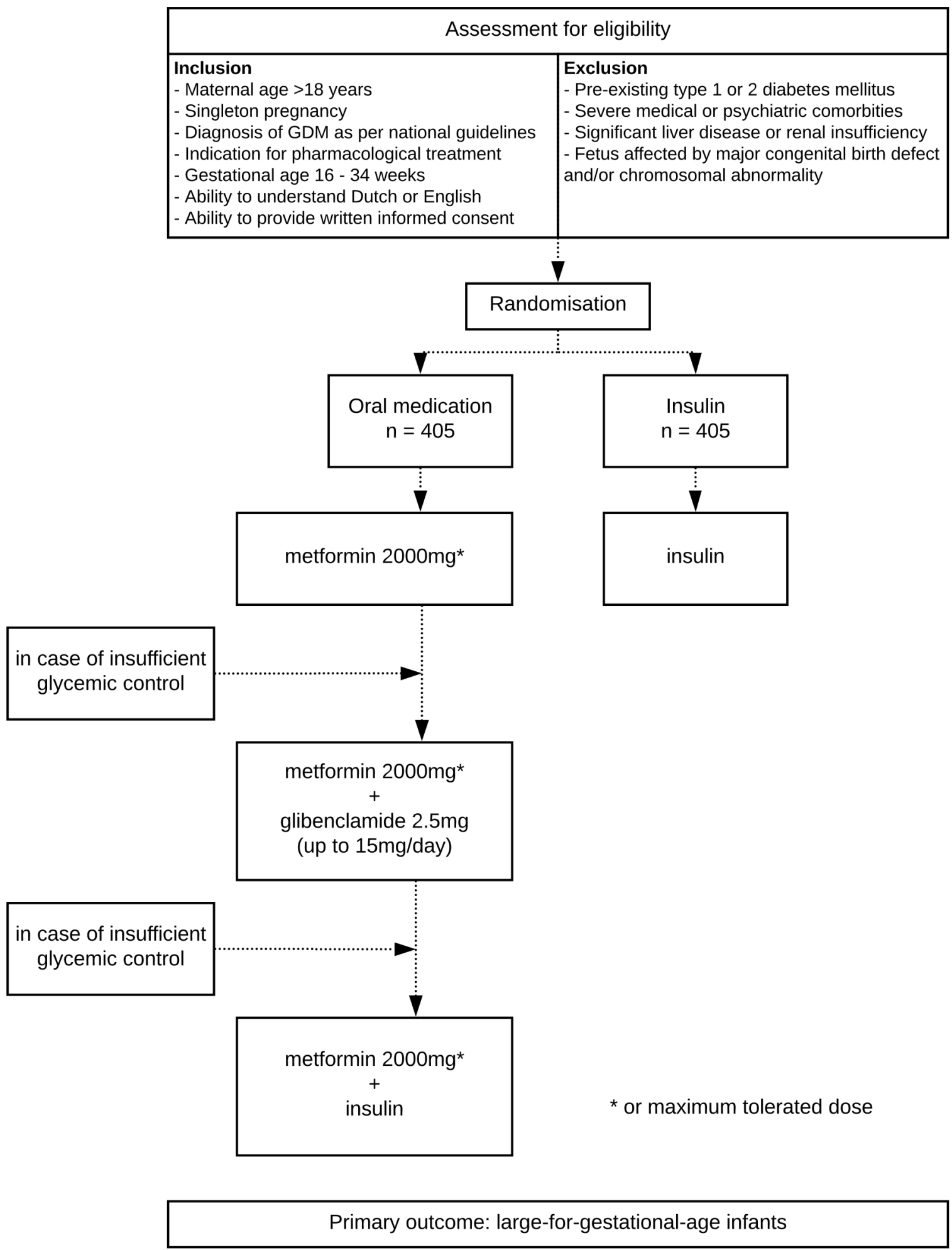

Figure 1 Flowchart of comparator (oral glucose lowering drugs) versus control (insulin). GDM, gestational diabetes mellitus. 
Oral glucose lowering drugs

In women allocated to the OGLD strategy, metformin is initiated with a starting dose of $500 \mathrm{mg}$ once daily for 3 days, followed by an increase of $500 \mathrm{mg}$ every 3 days to the final daily dose of $2000 \mathrm{mg}$ divided into two doses. In case of serious side effects (eg, severe nausea, persistent vomiting or diarrhoea), the metformin dose can be lowered to the maximum dose tolerated with acceptable side effects. Participants are advised to take metformin during or shortly after a meal to reduce side effects. In case of insufficient glycaemic control with metformin at the maximum (tolerated) dose, glibenclamide will be added at a starting dose of $2.5 \mathrm{mg}$ once daily. Glibenclamide can be increased if glycaemic goals are not met with increments of $2.5 \mathrm{mg}$ every week, up to a maximum dose of $15 \mathrm{mg}$ daily. In case of insufficient glycaemic control with both metformin and glibenclamide at the maximum doses, glibenclamide will be discontinued and replaced by insulin, while metformin will be continued.

\section{Insulin}

Participants randomised to insulin treatment will receive insulin according to usual practice, that is, in incremental doses until glycaemic targets are met. ${ }^{41}$ This includes both short-acting and long-acting insulin.

\section{Study procedures}

\section{Diabetes care}

In all participants, a specialised diabetes nurse or internal medicine specialist will review glycaemic control every 1-2 weeks using the following target values for glucose, as measured by capillary glucose self-testing: fasting $\leq 5.3 \mathrm{mmol} / \mathrm{L}, 1$-hour postprandial $\leq 7.8 \mathrm{mmol} / \mathrm{L}$ and 2-hours postprandial $\leq 6.7 \mathrm{mmol} / \mathrm{L}$. If titration of medication requires more frequent feedback, participants will be given the option to contact their diabetes treatment specialist in between scheduled visits. All participants receive the usual instructions regarding hypoglycaemic events (glucose $<4.0 \mathrm{mmol} / \mathrm{L}$ ). A participant diary is used to document glucose values and medication use, and is reviewed at every visit. Frequency of self-monitoring will be discussed on an individual basis with the treating diabetes team. Weight is documented at study inclusion and at every subsequent visit. Blood sampling for glycated haemoglobin (HbA1c) is performed at study inclusion, at 30 weeks and at 36 weeks of pregnancy.

\section{Obstetric care}

All participants will receive obstetrical care based on usual practice for GDM requiring pharmacological therapy. This includes assessment of fetal biometry at weeks 26-28, 30-32 and 34-36 of pregnancy by measuring fetal abdominal circumference, femur length, head circumference, estimated fetal weight (Hadlock or similar) and amniotic fluid volume. The timing of delivery follows local protocol, based on national guidelines. ${ }^{18}$ Induction of labour around 38-39 weeks of gestation is generally recommended for women with GDM requiring medication. Both oral antihyperglycaemic agents and insulin may be discontinued on the day of delivery in case of induced labour or as soon as labour is established after spontaneous onset. Monitoring of glucose levels during labour is advised.

\section{Neonatal care}

Neonatal glucose monitoring will be performed serially for up to 12-24 hours after delivery in accordance to local protocol in participating sites. We defined neonatal hypoglycaemia as a plasma glucose concentration $<2.6 \mathrm{mmol} / \mathrm{L}$ and severe neonatal hypoglycaemia as $<2.0 \mathrm{mmol} / \mathrm{L}$. ${ }^{42}$ Time and plasma glucose values are documented, as well interventions used, to regulate neonatal glucoses. Furthermore, any admission to a neonatal medium care or intensive care unit is documented.

\section{Postpartum}

Participants will attend routine obstetric and diabetes care provider appointments around 5-6 weeks postpartum at which time glucose self-monitoring will be carried out to detect persistent postpartum hyperglycaemia.

\section{Outcome measures}

Primary outcome measure

The primary outcome is a large-for-gestational-age (LGA) infant. LGA is defined as a birth weight $\geq 90$ th percentile using the Dutch Perinatal Registry reference charts. ${ }^{43}$

\section{Secondary outcome measures}

Secondary outcomes include maternal hypoglycaemia (biochemical hypoglycaemia $<3.9 \mathrm{mmol} / \mathrm{L}$, symptomatic hypoglycaemia, severe hypoglycaemia prompting the need for help by another person and/or hospital admission for hypoglycaemia), electiveand emergencycaesarean section, pregnancy-related hypertensive disorders including pregnancy-induced hypertension and PE, preterm delivery (delivery $<37$ weeks of gestation), postpartum neonatal hypoglycaemia (moderate: serum glucose $<2.6 \mathrm{mmol} / \mathrm{L}$, severe: serum glucose $<2.0 \mathrm{mmol} / \mathrm{L}$ ), neonatal hyperbilirubinaemia requiring phototherapy, neonatal medium care or intensive care admission and a cost-effectiveness analysis. These secondary outcomes were selected based on their clinical relevance and/or observed differences in previous studies comparing OGLDs and insulin.

Furthermore, a number of maternal baseline characteristics, additional obstetric- and neonatal outcomes, diabetes-related endpoints, biomarkers and laboratory examinations will be assessed (see online supplementary file 1 and 2).

\section{Follow-up}

Details regarding outcomes, including maternal and neonatal hospital admissions or complications, are recorded up to 6 weeks postpartum. Long-term follow-up of mother and child is not part of the initial trial; however, participants will be informed about planned long-term follow-up and asked to provide additional personal 
information and contact details on the patient information and informed consent form at study inclusion.

\section{Patient perspective and treatment satisfaction}

Side effects will be monitored using a custom-made form consisting of a short list of the most common side effects and the possibility to self-report any other experienced undesirable effects. The form will also address the actions taken as a response to side effects. Both treatment arms receive the same side effect form. Furthermore, treatment satisfaction is measured around 36 weeks of pregnancy using the Diabetes Treatment Satisfaction Questionnaire (DTSQ), consisting of eight questions regarding diabetes treatment and patient experience. ${ }^{44} 45$ Two additional questions regarding the impact of side effects and discomfort were provided by the copyright holder from a related treatment satisfaction measure for another condition, and added as items 9 and 10 of the DTSQ, to be analysed separately. ${ }^{46}$

\section{Safety and monitoring}

An independent Data and Safety Monitoring Board will be established to safeguard the interests of trial participants, assess the safety and efficacy of the interventions during the trial period and monitor the overall conduct of the clinical trial. An interim safety review is planned at 300 included participants and will be carried out by an independent statistician.

All serious adverse events (SAEs) reported by the subject or observed by the investigator or staff will be recorded. SAE definitions and standards for expedited reporting follow the International Council for Harmonisation of Technical Requirements for Registration of Pharmaceuticals for Human Use (ICH) Good Clinical Practice (GCP) guidelines on safety reporting. ${ }^{47}$ All SAEs will be reported to the accredited ethics committee that approved the protocol, according to the requirements of that committee.

\section{Sample size}

The primary outcome measure, rate of LGA infants, is anticipated to occur in $20 \%$ of patients in both study groups, based on a Dutch study cohort. ${ }^{48}$ We have set the non-inferiority limit at $8 \%$, which is equivalent to excluding a relative risk in the OGLD treatment compared with conventional insulin-based therapy greater than 1.4. With a one-sided significance level $(\alpha)$ of 0.025 and a power of 0.8 , the sample size is calculated at 393 patients in each arm. Accounting for a loss to follow-up of $3 \%, 810$ patients are needed (405 per arm).

\section{Analyses and reporting of results}

Primary and secondary outcomes

Primary analysis of the RCT results will be according to the intention-to-treat principle. Missing data will be handled according to the complete-case analysis principle, based on the availability of the components needed to determine the primary endpoint. Results will be reported according to Consolidated Standards of Reporting Trials guidelines, using the extension for non-inferiority trials. In case of substantial crossover $(>5 \%)$, a per protocol analysis is used additionally to the intention-to-treat analysis. Crossover is defined as patients not receiving the treatment allocated by randomisation (eg, participant never started treatment, treatment is no longer necessary for instance due to improved dietary adaptations, side effects or stopping treatment shortly after randomisation).

For the primary analysis, the non-inferiority of metformin/glibenclamide versus insulin for preventing LGA infants will be established when the upper bounds of the two-sided $95 \%$ CI for the risk ratio is less than 1.4. LGA will be defined as birth weight $>90$ th percentile. ${ }^{43}$ Results for the primary outcome will also be presented as absolute and relative risks (along with 95\% CI) and numbers needed to treat (if applicable). Analyses will not be adjusted for any observed differences in baseline characteristics between the arms.

The secondary outcome measures will be analysed similar to the primary outcome measure. Categorical secondary outcomes will be assessed by comparing the event rates in the two groups using a $\chi^{2}$ test with a $p$ value of 0.05 and also by presenting absolute and relative risks. For continuous secondary outcomes, differences between groups will be assessed with the Student's t-test if the outcome is normally distributed and with a non-parametric Mann-Whitney U test if skewed. These outcomes will be presented per group as means with $\mathrm{SD}$, geometric means with $95 \%$ CI or as median with IQR, depending on distribution.

\section{Subgroup analyses}

Subgroup analyses will be performed for women with and without a history of GDM, a family history of diabetes mellitus (first and/or second degree relative), BMI (normal weight, overweight, obese), according to severity of GDM (fasting and 2-hour OGTT glucose value by various diagnostic criteria and cut-offs) and sex (neonate). Additionally, potential causes for treatment failure of metformin alone will also be explored. Within the patients receiving oral agents, the outcome rate will be compared between the patients whose blood glucose could be regulated by metformin alone and those patients who also required glibenclamide and even additional insulin. Patient characteristics between these groups will be compared to identify possible contributing factors to metformin treatment failure.

\section{Economic evaluation}

An economic evaluation will be conducted alongside the RCT according to guidelines issued by the National Healthcare Institute. ${ }^{49}$ The EuroQol questionnaire (EQ-5D-5L) for health status measures is used at time of study inclusion, 36 weeks of pregnancy and 4-6 weeks postpartum. ${ }^{50}$ Further Health Technology Assessment questionnaires are based on the iMTA PCQ (Productivity Cost Questionnaire) and MCQ (Medical Consumption Questionnaire), issued at 36 weeks of pregnancy and 
4-6 weeks postpartum. ${ }^{51}{ }^{52}$ The statistical analysis for the economic evaluation will be done according to the intention-to-treat principle. Missing data will be imputed using multiple imputation. If OGLDs are non-inferior to insulin as hypothesised, a cost minimisation analysis will be performed to investigate which intervention is associated with lower costs. If non-inferiority cannot be shown, a cost-effectiveness analysis will be performed. The costs will be analysed from both a societal (ie, healthcare costs, patient and family costs, and costs in other sectors) and healthcare perspective (ie, only healthcare costs). In the cost minimisation analysis, the differences in costs between OGLDs and insulin will be evaluated using linear multilevel regression models with adjustment for covariates and effect modifiers if necessary. Bootstrapping with stratification for centre will be done to estimate $95 \%$ CI around differences in costs. In the cost-effectiveness analysis, cost and effect differences will be estimated using seemingly unrelated regression analyses while adjusting for confounders and effect modifiers if necessary. Incremental cost-effectiveness ratios (ICERs) will be calculated by dividing the difference in mean total costs between the treatment groups by the difference in mean effects. Bootstrapping with stratification for centre will be used to estimate uncertainty surrounding the ICERs. Uncertainty surrounding the ICERs will be graphically presented on cost-effectiveness planes. Cost-effectiveness acceptability curves showing the probability that the intervention is cost-effective in comparison with usual care for a range of different ceiling ratios will also be estimated. ${ }^{53} \mathrm{~A}$ sensitivity analysis will be performed to investigate the robustness of the results to variation in the most influential cost parameters such as medication and time required for clinical consults.

\section{Data handling}

Baseline data including patient demographics, obstetric and medical history, details regarding the pregnancy, delivery outcomes and diabetes treatment will be recorded using a web-based electronic case record form (eCRF) using Castor EDC. The eCRF is based on a standardised piloted eCRF that has been used in other multicentre trials within the NVOG Consortium 2.0 network and will be filled in by trained research nurses. The full eCRF is provided as online supplementary file 2. A study monitor will periodically visit participating centres, assessing quality of data and auditing trial conduct. Patient privacy will be ensured by allocation of unique participant numbers, which will be used on all study documentation. The participant code is only available to the local investigator and research staff.

\section{Ethics and dissemination}

This trial has been approved by the Medical Research Ethics Committee (MREC) of the UMC Utrecht. Trial reference number: 16-523/G-M-X. The MREC of the UMC Utrecht is accredited by the Central Committee on Research Involving Human Subjects (CCMO) since
November 1999. For all participating hospitals and study sites, approval by the boards of management will be obtained. The CCMO has issued a 'No grounds for non-acceptance' for the SUGAR-DIP trial. Research with a medicinal product must undergo an extra, marginal review alongside the review by the reviewing party (MREC). The competent authority (CCMO) checks if there are 'motivated objections' against the study. For this the European adverse reactions database (EudraVigilance) is checked for any previously reported suspected adverse reactions to the medicinal product, which could lead to unacceptable risks to the participating research subject. Furthermore, the CCMO is responsible as the competent authority for entering data into the European EudraCT database. EudraCT number for this trial: 2016-001401-16.

Changes to the study protocol are documented in amendments. Amendments are submitted for approval to the MREC. Major changes will be updated on the trial registration website. ${ }^{39}$ The full study protocol, including amendments, is publicly available on the study website. ${ }^{54}$

After completion of the trial, the principal investigator will report on the results of the main study and submit a manuscript to a peer-reviewed medical journal. Supplementary analyses will be reported separately.

\section{Author affiliations}

${ }^{1}$ Department of Obstetrics and Gynaecology, University Medical Center Utrecht, Utrecht University, Utrecht, The Netherlands

${ }^{2}$ Department of Obstetrics and Gynaecology, Amsterdam University Medical Centers, University of Amsterdam, Amsterdam, The Netherlands

${ }^{3}$ Department of Obstetrics and Gynaecology, Albert Schweitzer Hospital, Dordrecht, The Netherlands

${ }^{4}$ Department of Internal Medicine, Albert Schweitzer Hospital, Dordrecht, The Netherlands

${ }^{5}$ Department of Endocrinology and Metabolism, Amsterdam University Medical Centers, University of Amsterdam, Amsterdam, The Netherlands

${ }^{6}$ Department of Obstetrics and Gynaecology, Bernhoven Hospital, Uden, The Netherlands

${ }^{7}$ Department of Internal Medicine, Bernhoven Hospital, Uden, The Netherlands ${ }^{8}$ Department of Obstetrics and Gynaecology, Canisius Wilhelmina Hospital, Nijmegen, The Netherlands

${ }^{9}$ Department of Internal Medicine, Canisius Wilhelmina Hospital, Nijmegen, The Netherlands

${ }^{10}$ Department of Obstetrics and Gynaecology, Catharina Hospital, Eindhoven, The Netherlands

${ }^{11}$ Department of Internal Medicine, Catharina Hospital, Eindhoven, The Netherlands

${ }^{12}$ Department of Obstetrics and Gynaecology, Deventer Hospital, Deventer, The Netherlands

${ }^{13}$ Department of Internal Medicine, Deventer Hospital, Deventer, The Netherlands

${ }^{14}$ Department of Obstetrics and Gynaecology, Diakonessenhuis Utrecht, Utrecht, The Netherlands

${ }^{15}$ Department of Internal Medicine, Diakonessenhuis Utrecht, Utrecht, The Netherlands

${ }^{16}$ Department of Obstetrics and Prenatal Medicine, Erasmus MC University Medical Center Rotterdam, Rotterdam, The Netherlands

${ }^{17}$ Department of Obstetrics and Gynaecology, Flevoziekenhuis, Almere, The Netherlands

${ }^{18}$ Department of Internal Medicine, Flevoziekenhuis, Almere, The Netherlands

${ }^{19}$ Department of Obstetrics and Gynaecology, Gelderse Vallei Hospital, Ede, The Netherlands

${ }^{20}$ Department of Internal Medicine, Gelderse Vallei Hospital, Ede, The Netherlands

${ }^{21}$ Department of Obstetrics and Gynaecology, Gelre Hospitals, Apeldoorn, The Netherlands 
${ }^{22}$ Department of Internal Medicine, Gelre Hospitals, Apeldoorn, The Netherlands

${ }^{23}$ Department of Obstetrics and Gynaecology, Groene Hart Hospital, Gouda, The Netherlands

${ }^{24}$ Department of Internal Medicine, Groene Hart Hospital, Gouda, The Netherlands

${ }^{25}$ Department of Obstetrics and Gynaecology, Haaglanden Medical Center, The Hague, The Netherlands

${ }^{26}$ Department of Internal Medicine, Haaglanden Medical Center, The Hague, The Netherlands

${ }^{27}$ Department of Internal Medicine, Medical Center Jan van Goyen, Amsterdam, The Netherlands

${ }^{28}$ Department of Obstetrics and Gynaecology, Jeroen Bosch Hospital,

's-Hertogenbosch, The Netherlands

${ }^{29}$ Department of Internal Medicine, Jeroen Bosch Hospital, 's-Hertogenbosch, The Netherlands

${ }^{30}$ Department of Obstetrics and Gynaecology, Leiden University Medical Center, Leiden, The Netherlands

${ }^{31}$ Department of Medicine, Leiden University Medical Center, Leiden, The

Netherlands

${ }^{32}$ Department of Obstetrics and Gynaecology, Máxima Medical Center, Veldhoven, The Netherlands

${ }^{33}$ Department of Internal Medicine, Máxima Medical Center, Veldhoven, The Netherlands

${ }^{34}$ Department of Internal Medicine, Meander Medical Center, Amersfoort, The Netherlands

${ }^{35}$ Department of Obstetrics and Gynaecology, OLVG, Amsterdam, The Netherlands

${ }^{36}$ Department of Internal Medicine, OLVG, Amsterdam, The Netherlands

${ }^{37}$ Department of Obstetrics and Gynaecology, Radboud University Medical Center Nijmegen, Nijmegen, The Netherlands

${ }^{38}$ Department of Internal Medicine, Radboud University Medical Center Nijmegen,

Nijmegen, The Netherlands

${ }^{39}$ Department of Obstetrics and Gynaecology, Reinier de Graaf Hospital, Delft, The Netherlands

${ }^{40}$ Department of Internal Medicine, Reinier de Graaf Hospital, Delft, The Netherlands

${ }^{41}$ Department of Obstetrics and Gynaecology, Rijnstate Hospital, Arnhem, The Netherlands

${ }^{42}$ Department of Internal Medicine, Rijnstate Hospital, Arnhem, The Netherlands

${ }^{43}$ Department of Obstetrics and Gynaecology, Franciscus Gasthuis and Vlietland,

Rotterdam, The Netherlands

${ }^{44}$ Department of Internal Medicine, Franciscus Gasthuis and Vlietland, Rotterdam, The Netherlands

${ }^{45}$ Department of Obstetrics and Gynaecology, Spaarne Gasthuis, Haarlem, The Netherlands

${ }^{46}$ Department of Internal Medicine, Spaarne Gasthuis, Haarlem, The Netherlands

${ }^{47}$ Department of Obstetrics and Gynaecology, Tergooi, Blaricum, The Netherlands

${ }^{48}$ Department of Internal Medicine, Tergooi, Blaricum, The Netherlands

${ }^{49}$ Department of Obstetrics and Gynaecology, Amsterdam UMC, VU University

Medical Center, Amsterdam, The Netherlands

${ }^{50}$ Department of Internal Medicine, Amsterdam UMC, VU University Medical Center, Amsterdam, The Netherlands

${ }^{51}$ Department of Obstetrics and Gynaecology, Dijklander Hospital, Hoorn, The Netherlands

${ }^{52}$ Department of Internal Medicine, Dijklander Hospital, Hoorn, The Netherlands

${ }^{53}$ Department of Health Sciences, Faculty of Science, VU University Amsterdam, Amsterdam, The Netherlands

${ }^{54}$ Department of Obstetrics and Gynaecology, Meander Medical Center, Amersfoort, The Netherlands

${ }^{55}$ Department of Obstetrics and Gynaecology, School of Medicine, Monash

University, Melbourne, Australia, Melbourne, The Netherlands

${ }^{56}$ Department of Internal Medicine and Endocrinology, University Medical Center Utrecht, Utrecht University, Utrecht, The Netherlands

${ }^{57}$ Department of Neonatology, University Medical Center Utrecht, Utrecht University, Utrecht, The Netherlands

${ }^{58}$ Julius Center for Health Sciences and Primary Care, University Medical Center

Utrecht, Utrecht University, Utrecht, Netherlands

${ }^{59}$ Department of Internal Medicine, Amsterdam University Medical Centers,

University of Amsterdam, Amsterdam, The Netherlands

${ }^{60}$ Department of Obstetrics and Prenatal Medicine, Erasmus MC University Medical Center Rotterdam, Rotterdam, The Netherlands
Contributors Study concept, trial design and study protocol: LdW, DNV, JEB, IME, BWM, HWdV, FG, CAN, RP, JHD, AF, BBvR. Acquisition of data: LW, DR, BMCA, RMKK RCP, MRS, MALVD, FA, DHS, MARV, SMIK, MMO, JJZ, MJMD, TEV, PRJG, SG, WV, NH, TKK, RL, RH, AJMH, TB, CAvM, AWB, WH, SV, AGVV, RCD, HJJ, MS, EJPdK, JOEHL, PWP, IME, MESP, ESA, CBB, BBH, BJP, OWHvdH, BG, ML, JAW, KB, ACvB, FWM, SAE, MZ, WHvH, BAMBL, CRGMDG, MGAJW, RGIJ, NAMC, RZ. Analysis and interpretation of data: LdW, DR, DNV, JEB, IME, BWM, HWdV, FG, CAN, RCP, JHD, AF, BBvR. Drafting of the manuscript: LdW, DR, CAN, RCP, JHD, AF, BBvR. Critical revision of the manuscript for important intellectual content: LdW, DR, DNV, JEB, IME, BWM, HWV, FG, CAN, RCP, JHD, AF, BBvR, BMCA, RMKK, MRS, MALVD, FA, DHS, MARV, SMIK, MM0, JJZ, MJMD, TEV, PRJG, SG, WV, NH, TKK, RL, RH, AJMH, TB, CAvM, AWB, WH, SV, AGVV, RCD, HJJ, MS, EJPdK, JOEHL, PWP, MESP, ESA, CBB, BBH, BJP, OWHvdH, BG, ML, JAW, KB, ACvB, FWM, SAE, MZ, WHvH, BAMBL, CRGMDG, MGAJW, RGIJ, NAMC, RZ. Study supervision: JHD, AF, BBvR.

Funding The SUGAR-DIP trial and this work is investigator-driven and was supported by ZonMw (The Netherlands Organisation for Health Research and Development, the Hague), grant 80-83600- 98-40001.

Competing interests JHD sits on advisory boards for Novo Nordisk A/S. BWM is supported by a National Health and Medical Research Council Practitioner Fellowship (GNT1082548). BWM reports consultancy for ObsEva, Merck KGaA and Guerbet.

Provenance and peer review Not commissioned; externally peer reviewed.

Open access This is an open access article distributed in accordance with the Creative Commons Attribution Non Commercial (CC BY-NC 4.0) license, which permits others to distribute, remix, adapt, build upon this work non-commercially, and license their derivative works on different terms, provided the original work is properly cited, appropriate credit is given, any changes made indicated, and the use is non-commercial. See: http://creativecommons.org/licenses/by-nc/4.0/.

\section{REFERENCES}

1. Jiwani A, Marseille E, Lohse N, et al. Gestational diabetes mellitus: results from a survey of country prevalence and practices. J Matern Fetal Neonatal Med 2012;25:600-10.

2. Guariguata L, Linnenkamp U, Beagley J, et al. Global estimates of the prevalence of hyperglycaemia in pregnancy. Diabetes Res Clin Pract 2014;103:176-85.

3. Zhu Y, Zhang C. Prevalence of Gestational Diabetes and Risk of Progression to Type 2 Diabetes: a Global Perspective. Curr Diab Rep 2016;16:7.

4. Buckley BS, Harreiter J, Damm P, et al. Gestational diabetes mellitus in Europe: prevalence, current screening practice and barriers to screening. A review. Diabet Med 2012;29:844-54.

5. Wendland EM, Torloni MR, Falavigna M, et al. Gestational diabetes and pregnancy outcomes--a systematic review of the World Health Organization (WHO) and the International Association of Diabetes in Pregnancy Study Groups (IADPSG) diagnostic criteria. BMC Pregnancy Childbirth 2012;12:23.

6. Metzger BE, Lowe LP, Dyer AR, et al. Hyperglycemia and adverse pregnancy outcomes. N Engl J Med 2008;358:1991-2002.

7. Xiong X, Saunders LD, Wang FL, et al. Gestational diabetes mellitus: prevalence, risk factors, maternal and infant outcomes. Int J Gynaecol Obstet 2001;75:221-8.

8. Voormolen DN, de Wit L, van Rijn BB, et al. Neonatal Hypoglycemia Following Diet-Controlled and Insulin-Treated Gestational Diabetes Mellitus. Diabetes Care 2018;41:1385-90.

9. Buchanan TA, Xiang AH, Page KA. Gestational diabetes mellitus: risks and management during and after pregnancy. Nat Rev Endocrinol 2012;8:639-49

10. Scholtens DM, Kuang A, Lowe LP, et al. Hyperglycemia and Adverse Pregnancy Outcome Follow-up Study (HAPO FUS): Maternal Glycemia and Childhood Glucose Metabolism. Diabetes Care 2019;42:381-92

11. Malcolm J. Through the looking glass: gestational diabetes as a predictor of maternal and offspring long-term health. Diabetes Metab Res Rev 2012;28:307-11.

12. Tam WH, Ma RCW, Ozaki R, et al. In Utero Exposure to Maternal Hyperglycemia Increases Childhood Cardiometabolic Risk in Offspring. Diabetes Care 2017;40:679-86.

13. Crowther CA, Hiller JE, Moss JR, et al. Effect of treatment of gestational diabetes mellitus on pregnancy outcomes. N Engl J Med 2005;352:2477-86

14. Landon MB, Spong CY, Thom E, et al. A multicenter, randomized trial of treatment for mild gestational diabetes. $N$ Engl J Med 2009;361:1339-48. 
15. The Royal Australian College of General Practitioners. General practice management of type 2 diabetes: 2016-18. East Melbourne, Vic: RACGP, 2016. https://www.racgp.org.au/clinical-resources/ clinical-guidelines/key-racgp-guidelines/view-all-racgp-guidelines/ management-of-type-2-diabetes/reproductive-health/gestationaldiabetes\#ref-num-65 (Accessed 27 Mar 2019).

16. Committee on Practice Bulletins-Obstetrics. ACOG Practice Bulletin No. 190: Gestational Diabetes Mellitus. Obs Gynecol 2018;131:e49-64.

17. American Diabetes Association. 13. Management of Diabetes in Pregnancy: Standards of Medical Care in Diabetes-2018. Diabetes Care 2018;41:S137-S143.

18. Nederlandse Vereniging Voor Obstetrie en Gynaecologie. DIABETES MELLITUS EN ZWANGERSCHAP Versie 2.0. $2010 \mathrm{http}: / / \mathrm{www}$. nvog-documenten.nl/index.php?pagina=/richtlijn/item/pagina.php\& richtlijn_id=863 (accessed 26 Oct 2016).

19. National Institute for Health and Care Excellence. Diabetes in pregnancy: management from preconception to the postnatal period. 2015:2-65 https://www.nice.org.uk/guidance/ng3 (accessed 8 Nov 2018).

20. Keely E, Berger H, Feig DS, et al. New Diabetes Canada Clinical Practice Guidelines for Diabetes and Pregnancy - What's Changed? Journal of Obstetrics and Gynaecology Canada 2018;40:1484-9.

21. Society of Maternal-Fetal Medicine Publications Committee. SMFM Statement: Pharmacological treatment of gestational diabetes. Am J Obs Gynecol 2018;218:B2-4.

22. Langer $\mathrm{O}$, Conway DL, Berkus MD, et al. A comparison of glyburide and insulin in women with gestational diabetes mellitus. N Engl $J$ Med 2000;343:1134-8.

23. Rowan JA, Hague WM, Gao W, et al. Metformin versus insulin for the treatment of gestational diabetes. N Engl J Med 2008;358:2003-15.

24. Langer $O$. Pharmacological treatment of gestational diabetes mellitus: point/counterpoint. Am J Obstet Gynecol 2018;218:490-9.

25. Hod M, Kapur A, Sacks DA, et al. The International Federation of Gynecology and Obstetrics (FIGO) Initiative on gestational diabetes mellitus: A pragmatic guide for diagnosis, management, and care. Int $J$ Gynaecol Obs 2015;131 Suppl:S173-211.

26. Camelo Castillo W, Boggess K, Stürmer T, et al. Trends in glyburide compared with insulin use for gestational diabetes treatment in the united states, 2000-2011. Obstetrics \& Gynecology 2014;123:1177-84

27. Brown J, Martis R, Hughes B, et al. Oral anti-diabetic pharmacological therapies for the treatment of women with gestational diabetes. Cochrane Database Syst Rev 2017:1:CD011967.

28. Brown J, Grzeskowiak L, Williamson K, et al. Insulin for the treatment of women with gestational diabetes. Cochrane Database Syst Rev 2017; 11:CD012037.

29. Figueroa Gray M, Hsu C, Kiel L, et al. 'It's a Very Big Burden on Me': Women's Experiences Using Insulin for Gestational Diabetes. Matern Child Health J 2017;21:1678-85.

30. Maymone AC, Baillargeon JP, Ménard J, et al. Oral hypoglycemic agents for gestational diabetes mellitus? Expert Opin Drug Saf 2011;10:227-38.

31. Ryu RJ, Hays KE, Hebert MF. Gestational diabetes mellitus management with oral hypoglycemic agents. Semin Perinatol 2014;38:508-15.

32. Latif $\mathrm{L}$, Hyer $\mathrm{S}$, Shehata $\mathrm{H}$. Metformin effects on treatment satisfaction and quality of life in gestational diabetes. $\mathrm{Br} J$ Diabetes Vasc Dis 2013;13:178-82.

33. Bradley C, Todd C, Gorton T, et al. The development of an individualized questionnaire measure of perceived impact of diabetes on quality of life: the ADDQoL. Qual Life Res 1999;8:79-91.

34. Balsells M, García-Patterson A, Solà I, et al. Glibenclamide, metformin, and insulin for the treatment of gestational diabetes: a systematic review and meta-analysis. BMJ 2015;350:h102.
35. Yogev $\mathrm{Y}$, Ben-Haroush A, Chen R, et al. Undiagnosed Asymptomatic Hypoglycemia. Obstetrics \& Gynecology 2004;104:88-93.

36. van Weelden W, Wekker V, de Wit L, et al. Long-Term Effects of Oral Antidiabetic Drugs During Pregnancy on Offspring: A Systematic Review and Meta-analysis of Follow-up Studies of RCTs. Diabetes Ther 2018;9:1811-29.

37. Nachum Z, Zafran N, Salim R, et al. Glyburide Versus Metformin and Their Combination for the Treatment of Gestational Diabetes Mellitus: A Randomized Controlled Study. Diabetes Care 2017;40:332-7.

38. Zorgevaluatie Nederland. Nederlandse Vereniging voor Obstetrie en Gynaecologie. http://www.zorgevaluatienederland.nl/associations/1 (Accessed 4 Feb 2019).

39. Netherlands Trial Register (NTR): The SUGAR-DIP trial; oral medication strategy versus insulin for diabetes in pregnancy. https:// www.trialregister.nl/trial/5953 (Accessed 29 Jan 2019).

40. Sénat MV, Affres $\mathrm{H}$, Letourneau A, et al. Effect of Glyburide vs Subcutaneous Insulin on Perinatal Complications Among Women With Gestational Diabetes: A Randomized Clinical Trial. JAMA 2018:319:1773-80.

41. Nederlandse Internisten Vereeniging (NIV). Diabetes en Zwangerschap. 2007.

42. Cornblath M, Hawdon JM, Williams AF, et al. Controversies regarding definition of neonatal hypoglycemia: suggested operational thresholds. Pediatrics 2000;105:1141-5.

43. Visser GH, Eilers PH, Elferink-Stinkens PM, et al. New Dutch reference curves for birthweight by gestational age. Early Hum Dev 2009;85:737-44.

44. Bradley C, Lewis KS. Measures of psychological well-being and treatment satisfaction developed from the responses of people with tablet-treated diabetes. Diabet Med 1990;7:445-51.

45. Bradley C. Handbook of psychology and diabetes: a guide to psychological measurement in diabetes research and practice. Chur, Switzerland: Harwood Academic Publishers, 1994.

46. Barendse SM, Speight J, Bradley C. The Renal Treatment Satisfaction Questionnaire (RTSQ): a measure of satisfaction with treatment for chronic kidney failure. Am J Kidney Dis 2005;45:572-9.

47. Baber N. International conference on harmonisation of technical requirements for registration of pharmaceuticals for human use $(\mathrm{ICH})$. Br J Clin Pharmacol 1994;37:401-4 https://www.ich.org/fileadmin/ Public_Web_Site/ICH_Products/Guidelines/Efficacy/E2A/Step4/E2A Guideline.pdf.

48. Lamain-de Ruiter M, Kwee A, Naaktgeboren CA, et al. External validation of prognostic models to predict risk of gestational diabetes mellitus in one Dutch cohort: prospective multicentre cohort study. BMJ 2016;354:14338.

49. Zorginstituut Nederland. Richtlijn Voor Het Uitvoeren van Economische Evaluaties in de Gezondheidszorg. Diemen 2015

50. Herdman M, Gudex C, Lloyd A, et al. Development and preliminary testing of the new five-level version of EQ-5D (EQ-5D-5L). Qual Life Res 2011;20:1727-36.

51. Bouwmans C, Hakkaart- van Roijen L, Koopmanschap M, et al. Manual iMTA Productivity Cost Questionnaire (iPCQ). Rotterdam: Institute for Medical Technology Assessment Erasmus Universiteit Rotterdam, 2013.

52. Bouwmans C, Hakkaart- van Roijen L, Koopmanschap M, et al. Manual iMTA Medical Consumption Questionnaire (iMCQ). Rotterdam: Institute for Medical Technology Assessment Erasmus Universiteit Rotterdam, 2013.

53. Fenwick E, O'Brien BJ, Briggs A. Cost-effectiveness acceptability curves-facts, fallacies and frequently asked questions. Health Econ 2004;13:405-15.

54. Zorgevaluatie Nederland. Zorgevaluatie Nederland Study website: SUGAR-DIP trial. http://www.zorgevaluatienederland.nl/projects/29/ downloads-and-links (Accessed 4 Feb 2019). 